\section{The Recombination Law for Weak Ionization}

In order to explain the observed relation between the number of condensation nuclei in atmospheric air and the ionization, an equilibrium equation was proposed $^{1}$ of the type $q=a n+b n Z$, where $q$ is the rate of production of ion-pairs per om., $n$ and $Z$ the concentrations of small ions (of one sign) and nuclei, and $a$ and $b$ are constants for any given conditions. This implies that, in atmospheric air, the rate of loss of ions by recombination among themselves is proportional to $n$ and not to $n^{2}$. It was suggested that, where a volume of air is traversed at any time by only a few ionizing particles, the rate of loss of ions from the space by recombination is proportional to the number of ion tracks and hence approximately to $n$.

To test this view, experiments have been carried out on air free from nuclei contained in a vessel of volume 730 litres. The intensity of the ionization inside the vessel was varied by placing a small quantity of radium at different distances from it. Observations were made of $n$ for different values of $q$. In experiments of this kind, ions are lost by diffusion as well as by recombination, and the chief dificulty is to make a proper allowanee for this loss. In this work, correction for diffusion has been made on the lines developed by Power ${ }^{2}$, and it is believed that the corrected values of $n$ are fairly accurate.

Using corrected values of $n$, and plotting log $n$ against $\log q$, we obtain a graph the slope of which varies. For values of $q$ between 360 and 120 , the slope is 2.0 , in agreement with the ordinary law $q=\alpha n^{2}$. For lower values of $q$ the slope becomes less. For values of $q$ between 30 and 12, it is approximately 1.45 . Experiments in the range $q<12$ have not as yet been possible. Putting the law in the form $q=k n^{p}$, the experiments indicate a considerable diminution in the value of $p$ over the range of observation. It is possible that with lower values of $q, p$ may approach to unity, giving the linear recombination law proposed to explain the equilibrium of ionization in the atmosphere.

It is perhaps worthy of note that in the region where the law $q=\alpha n^{2}$ appears to hold, the calculated value of $\alpha$ is $1.56 \times 10^{-6}$, which is closer to the values obtained by the earlier workers than to the more recent values such as that of Sayers ${ }^{3}$.

\section{University College,}

Nolan, J. J., Proc. Royal Irish Acad., 46, 77 (1940).

2 Power, A. D., J. Frank. Inst., 96, 327 (1923).

3 Sayers, J., Proe. Roy. Soc., A, 169, 83 (1938).

\section{Surface Films of Polar Crystals}

THE study of atomic monolayers was initiated by Miss Pockels and the late Lord Rayleigh long ago, for capillary surfaces. It was capable of extension to electric crystalline structure, on the basis of the analysis of local polarization which is to be found in Maxwell's "Treatise" but is usually quoted under the names of Clausius and Lorentz. It compels the result that for a erystal polarized transverse to its surface a monolayer of compensating ions is required on its face, but in number half that in each ionic sheet of the crystal ${ }^{1}$.

I have looked for a long time for some confirmation of so remarkable a result. The fundamental dis- covery of the diffraction of uniform pencils of electrons at the surface of a crystal, independently by Davisson and Germer in New York and by G. P. Thomson and A. Reid in Aberdeen, gave promise in this direction. This subject has now grown into an immense technical science with which only experts can be familiar, as is illustrated in the recent treatise by G. P. Thomson and W. Cochrane ${ }^{2}$. In turning over its pages I have hit upon the confirmation which I wanted, relating to the surface film, in a passage (p. 270) here reproduced, on the diffraction of pencils of slow electrons. It describes results reported in Davisson and Germer's early memoir.

"The new beams . . . thus possess the character of plane- rather than space-grating beams. Measurement shows, however, that the plane-grating spacing to which they correspond is in each case exactly twice that of the nickel atoms in the azimuth in question."

I have, however, not succeeded in explaining the coneept of an index of refraction of the electrons along such lines.

\section{Holywood,}

JOSEPH LARMOR.

Northern Ireland. May 12.

${ }^{1}$ Cf. Larmor, "Mathematical and Physical Papers", 2, 620-29 (1928), "On Electro-crystalline Properties as conditioned by Atomic Lattices" reprinted from Proc. Roy. Soc., A, 99 (1921), following on earlier discussions in Phil. Trans., 2, 44-50 (1897). 2 "Theory and Practice of Electron Diffraction" (Macmillan, 1939).

\section{Flow Properties of Some Thermoplastics}

Scott Blair and Coppen ${ }^{1}$, dealing with the compression of cylinders under constant stress, have given a general method of specifying the rheological properties of plastic materials by making use of two characteristics $\psi, k$ given by

$$
\psi=s \sigma^{-1} t k
$$

where $s, \sigma, t$ have their usual meaning. Broome and Bilmes ${ }^{2}$ have confirmed the validity of the treatment by their work on the stretching of asphalt strips.

Scott Blair ${ }^{3}$ had previously suggested a modification of the Bingham-Murray equation for the empty. ing of an initially full capillary tube, instead of the usual filling of an empty one. For the case of materials not obeying Poiseuille's law, he suggested a parabolic plot of the data obtained. Fundamentally, as applied to the original Bingham-Murray method, this modification can be expressed by

$$
\Psi=\frac{P R^{2}}{4} \cdot \frac{1}{h^{2}-h_{0}{ }^{2}} t^{K},
$$

where $P$ is the pressure, $R$ the radius of eapillary, $h$ the length of extrusion, $t$ the time, and $h_{0}$ is the extrusion at time $t=0$, and the exponent $K$ "differs from unity to an extent which is the measure of the discrepancy from truly fluid behaviour". When $K=1, \Psi$ corresponds to a true viscosity. There is a formal similarity between equations (1) and (2).

The value of the stress averaged across a diameter of the tube is $P R / 4 h$. From a comparison of the differential form of the Bingham-Murray equation

$$
\overline{d t}\left[\frac{2 h}{R}\right]=\frac{1}{\eta} \frac{P R,}{4 h} \quad . \quad . \quad .
$$

with the equation defining the viscosity, $\eta_{i}$,

$$
\frac{d \sigma}{d t}=\frac{1}{\eta} s
$$

we see that $2 h / R$ can be regarded as the value of the strain averaged across a diameter. Using this ex- 